\title{
AN UPPER BOUND ON JACOBSTHAL'S FUNCTION
}

\author{
FINTAN COSTELLO AND PAUL WATTS
}

\begin{abstract}
The function $h(k)$ represents the smallest number $m$ such that every sequence of $m$ consecutive integers contains an integer coprime to the first $k$ primes. We give a new computational method for calculating strong upper bounds on $h(k)$.
\end{abstract}

\section{INTRODUCTION}

Letting $p_{i}$ be the $i$ th prime and $P_{k}$ be the product of the all primes up to $p_{k}$, Jacobsthal's function $h(k)$ represents the smallest number $m$ such that every sequence of $m$ consecutive integers contains an integer coprime to $P_{k}$. The function $h(k)$ has been studied by a number of different authors, and is central to results on the maximal gaps between consecutive primes [7, [8] and on the least prime in arithmetic progressions [9]. Explicit values of $h(k)$ are known only for $k \leq 49$, with the computation of $h(49)$ by Hagedorn taking two months on a cluster of 30 computers [2].

Let $g(n)$ represent the smallest number $m$ such that every sequence of $m$ consecutive integers contains an integer coprime to $n$. In a letter to Erdös [1], Jacobsthal asked whether

$$
\begin{aligned}
& h(k) \leq C k^{2}, \\
& g(n) \leq h(k) \text { for all } n \text { with } k \text { distinct prime factors }
\end{aligned}
$$

both hold for all positive $k$ and some constant $C$. Iwaniec's proof [5] that

$$
h(k) \leq C(k \log k)^{2}
$$

for an unknown constant $C$ gives our closest approach to the first of these conjectures. Hajdu and Saradha 44 recently disproved the second conjecture using the explicit values of $h(k)$ calculated by Hagedorn.

The best known explicit upper bounds on $h(k)$, of

$$
\begin{aligned}
& h(k) \leq 2^{k}, \\
& h(k) \leq 2 k^{2+2 e \log k},
\end{aligned}
$$

are due to Kanold [6] and Stevens [10] respectively (with the second bound being stronger for $k \geq 260$ ). These bounds are quite weak: while from Hagedorn's calculations we have $h(49)=742$, these bounds give $h(49)<10^{15}$ and $h(49)<10^{40}$ respectively. We thus know relatively little about the explicit behaviour of $h(k)$ for $k$ greater than 49 .

Received by the editor May 24, 2012 and, in revised form, September 16, 2013 and September $26,2013$.

2010 Mathematics Subject Classification. Primary 11N25; Secondary 11Y55. 
In this paper we address this gap using a new computational method for computing explicit upper bounds on $h(k)$. This method gives bounds much stronger than those given by Kanold and Stevens; for example, this method gives a bound on $h(49)$ which is less than 3 times the true value of $h(49)$. This method is also much faster than that used by Hagedorn to calculate values of $h(k)$, computing a bound on $h(49)$ in seconds. We used this method to compute upper bounds on $h(k)$ for $k$ from 50 to 10,000: for all $k$ in that range we find

$$
h(k) \leq 0.27749612254 k^{2} \log k
$$

a bound hundreds of orders of magnitude stronger than those given by Kanold and Stevens in this range.

Our method is based on an expression for the function $\pi_{\min }(m, k)$, which represents the smallest number $x$ such that every sequence of $m$ consecutive integers contains at least $x$ integers coprime to $P_{k}$. Taking $\phi(\cdot)$ to be Euler's totient function, $\pi_{\min }(m, k)$ has the recurrent lower bound

$$
\begin{aligned}
\phi\left(P_{k}\right)\left\lfloor\frac{m}{P_{k}}\right\rfloor+r-\sum_{i=1}^{k}\left\lceil\frac{r}{p_{i}}\right\rceil & +\sum_{i=2}^{k}\left\lfloor\frac{r}{2 p_{i}}\right\rfloor+E \\
& +\sum_{i=2}^{k-1} \sum_{j=i+1}^{k} \pi_{\min }\left(\left\lfloor\frac{r}{p_{i} p_{j}}\right\rfloor, i-1\right) \leq \pi_{\min }(m, k)
\end{aligned}
$$

where $r=m \bmod P_{k}$ and where $E$ is a positive correction arising due to constraints on the co-occurence of residues of the primes up to $p_{k}$. This expression has the computationally nice property that the recurrent double sum is dominated by terms where $i$ is small, and putting computational effort into calculating accurate values for $\pi_{\min }(r, i-1)$ across all candidate $r$ 's for some set of low values of $i$ allows us to efficiently compute strong lower bounds on $\pi_{\min }(m, k)$ for a large range of values of $k$. Since it is clear that

$$
0<\pi_{\min }(m, k) \Rightarrow h(k) \leq m,
$$

this in turn allows us to efficiently compute strong upper bounds on $h(k)$ across a large range of $k$.

In the first three parts of this paper we prove this expression. In the last part we describe algorithms based on this expression that we use to compute explicit upper bounds on $h(k)$.

\section{Preliminaries}

We take $\omega(a)$ to represent the number of distinct prime factors of $a, \omega_{k}(a)$ to be the number of distinct primes that are factors of both $a$ and $P_{k}$, and $l_{k}(a)$ to be the lowest factor of $a$ that is also a factor of $P_{k}$.

For any set of integers $S$ we take $F_{S}(d)$ to be the number of integers in $S$ that are divisible by $d$; for any integer $b$ we take $F_{b, m}(d)$ to be the number of integers in the sequence of $m$ consecutive integers $b+1, \ldots, b+m$ that are divisible by $d$. We take $\pi(b, m, i)$ to be the number of integers in the sequence $b+1, \ldots, b+m$ that are coprime to $P_{i}$, and take $\pi_{\min }(m, i)$ to be the lowest value of $\pi(b, m, i)$ across all $b$.

We use the following result concerning divisors of members of arithmetic sequences. 
Theorem 2.1. Let $d$ and $n$ be two coprime squarefree integers. Then for any arithmetic sequence

$$
b+d, b+2 d, \ldots
$$

there necessarily exists a corresponding sequence of consecutive integers

$$
c b+1, c b+2, \ldots
$$

such that

$$
\operatorname{GCD}(c b+x, n)=\operatorname{GCD}(b+x d, n) \text { for all } x .
$$

Proof. Choose integers $c$ and $z$ such that $c d-z n=1$. Since $c$ divides $z n+1$ it is clear that $c$ and $n$ are coprime and so we have

$$
\operatorname{GCD}(c(b+x d)-x z n, n)=\operatorname{GCD}(b+x d, n) \text { for all } x
$$

(because $x z n$ shares all of $n$ 's prime factors while the only prime factors that $c(b+$ $x d$ ) shares with $n$ are those of $(b+x d)$ ). Rearranging the left-hand side (and using the fact that $c d-z n=1$ ) we get

$$
c(b+x d)-x z n=c b+x(c d-z n)=c b+x \text { for all } x
$$

and the consecutive sequence starting at $c b+1$ has the required property.

(Hajdu and Saradha give a a similar result [3].) Following from this we define $c_{b}(d)$ as follows:

Definition 2.2. For some integer $d$ with $p_{i}$ as its lowest prime factor, let $y+d$ be the first term in the sequence $b+1, \ldots, b+m$ that has $d$ as a divisor. We have then an arithmetic sequence

$$
y+d, y+2 d, \ldots, y+F_{b, m}(d) \times d
$$

all of whose members are divisible by $d$ and all of which are in the sequence $b+$ $1, \ldots, b+m$. Then $c_{b}(d)$ is an integer such that

$$
\operatorname{GCD}\left(c_{b}(d)+x, P_{i-1}\right)=\operatorname{GCD}\left(y+x d, P_{i-1}\right) \text { for all } x
$$

and so the sequence of consecutive integers starting at $c_{b}(d)+1$ all have the same prime factors in common with $P_{i-1}$ as the corresponding terms in the arithmetic sequence of integers between $b+1$ and $b+m$ that have $d$ as a divisor.

\section{A RECURRENT EXPRESSION FOR $\pi(b, m, k)$}

We now prove a recurrent expression for $\pi(b, m, k)$, the number of integers from $b+1$ to $b+m$ which are coprime to $P_{k}$.

Theorem 3.1. For integers $m, b$ and $k$ we have

$$
\pi(b, m, k)=m-\sum_{i=1}^{k} F_{b, m}\left(p_{i}\right)+\sum_{\substack{b<a \leq b+m \\ \omega_{k}(a)>0}}\left(\omega_{k}(a)-1\right) .
$$

Proof. The expression

$$
m-\sum_{i=1}^{k} F_{b, m}\left(p_{i}\right)
$$


undercounts $\pi(b, m, k)$ by $\omega_{k}(a)-1$ for each integer $a$ in our sequence that has $\omega_{k}(a)>0$, and so

$$
\pi(b, m, k)=m-\sum_{i=1}^{k} F_{b, m}\left(p_{i}\right)+\sum_{\substack{b<a \leq b+m \\ \omega_{k}(a)>0}}\left(\omega_{k}(a)-1\right)
$$

as required.

Theorem 3.2. For any $p_{x} \mid P_{k}$ let $S$ be the set of integers a such that $b<a \leq b+m$ and $l_{k}(a)=p_{x}$. Then

$$
\sum_{a \in S}\left(\omega_{k}(a)-1\right)=\sum_{x<i \leq k} F_{S}\left(p_{i} p_{x}\right)
$$

Proof. Assume some $p_{x} \mid P_{k}$. For any $a \in S$ the total number of composites $p_{x} p_{i}$ dividing $a$ where $p_{i} \mid P_{k}$ is therefore equal to the number of prime factors $p_{i} \neq p_{x}$ of $P_{k}$ which divide $a$. Since $p_{x} \mid a$ this total is equal to $\omega_{k}(a)-1$. Each composite $p_{i} p_{x}$ thus contributes 1 to the sum

$$
\sum_{a \in S}\left(\omega_{k}(a)-1\right)
$$

for each $a \in S$ which has $p_{i} p_{x}$ as a divisor and so the total contribution that each such composite makes to that sum is $F_{S}\left(p_{i} p_{x}\right)$, and the result follows.

Theorem 3.3.

$$
\sum_{\substack{b<a \leq b+m \\ \omega_{k}(a)>0}}\left(\omega_{k}(a)-1\right)=\sum_{j=2}^{k} F_{b, m}\left(2 p_{j}\right)+\sum_{i=2}^{k-1} \sum_{j=i+1}^{k} \pi\left(c_{b}\left(p_{i} p_{j}\right), F_{b, m}\left(p_{i} p_{j}\right), i-1\right)
$$

Proof. The proof is inductive. For the base of the induction, we note from Theorem 3.2 that

$$
\sum_{\substack{b<a \leq b+m \\ \omega_{1}(a)>0}}\left(\omega_{k}(a)-1\right)=\sum_{j=2}^{k} F_{b, m}\left(2 p_{j}\right) .
$$

To prove induction we begin by assuming that

$$
\sum_{\substack{b<a \leq b+m \\ \omega_{x}(a)>0}}\left(\omega_{k}(a)-1\right)=\sum_{j=2}^{k} F_{b, m}\left(2 p_{j}\right)+\sum_{i=2}^{x} \sum_{j=i+1}^{k} \pi\left(c_{b}\left(p_{i} p_{j}\right), F_{b, m}\left(p_{i} p_{j}\right), i-1\right)
$$

holds for some $x<k-1$.

Let $S$ be the set of integers in our sequence $b+1, \ldots, b+m$ which are coprime to $P_{x}$ and which have $p_{x+1}$ as a factor. Since all integers not in $S$ have either already been counted or have $\omega_{x+1}(a)=0$, we have

$$
\sum_{\substack{b<a \leq b+m \\ \omega_{x+1}(a)>0}}\left(\omega_{k}(a)-1\right)=\sum_{\substack{b<a \leq b+m \\ \omega_{x}(a)>0}}\left(\omega_{k}(a)-1\right)+\sum_{a \in S}\left(\omega_{k}(a)-1\right) .
$$

By definition $l_{k}(a)=p_{x+1}$ for all $a \in S$, and so from Theorem 3.2 we have

$$
\sum_{a \in S}\left(\omega_{k}(a)-1\right)=\sum_{x+1<j \leq k} F_{S}\left(p_{j} p_{x+1}\right) .
$$


We can rewrite the right-hand side here as

$$
\sum_{x+1<i \leq k} F_{S}\left(p_{i} p_{x+1}\right)=\sum_{j=x+2}^{k} \sum_{\begin{array}{c}
b<a \leq b+m \\
p_{x+1} p_{j} \mid a \\
a \text { coprime to } P_{x}
\end{array}} 1 .
$$

For each pair $p_{x+1} p_{j}$ we have an arithmetic subsequence consisting of those integers between $b+1$ and $b+m$ that have $p_{x+1} p_{j}$ as a factor. This subsequence contains $F_{b, m}\left(p_{j} p_{x+1}\right)$ integers. The right-hand side in the above expression contributes 1 for each member of this arithmetic subsequence which is coprime to $P_{x}$. From Theorem 2.1 this arithmetic subsequence is equivalent to a sequence of $F_{b, m}\left(p_{j} p_{x+1}\right)$ consecutive integers starting at $c_{b}\left(p_{x+1} p_{j}\right)$, and so the right-hand side above contributes 1 for each member of this sequence which is coprime to $P_{x}$, giving a total contribution of

$$
\pi\left(c_{b}\left(p_{x+1} p_{j}\right), F_{b, m}\left(p_{j} p_{x+1}\right), x\right)
$$

for each such pair. We thus have

$$
\sum_{a \in S}\left(\omega_{k}(a)-1\right)=\sum_{j=x+2}^{k} \pi\left(c_{b}\left(p_{x+1} p_{j}\right), F_{b, m}\left(p_{j} p_{x+1}\right), x\right)
$$

and so

$$
\sum_{\substack{b<a \leq b+m \\ \omega_{x+1}(a)>0}}\left(\omega_{k}(a)-1\right)=\sum_{j=2}^{k} F_{b, m}\left(2 p_{j}\right)+\sum_{i=2}^{x+1} \sum_{j=x+2}^{k} \pi\left(c_{b}\left(p_{i} p_{j}\right), F_{b, m}\left(p_{i} p_{j}\right), i-1\right)
$$

also holds for $x+1$. This completes the induction and gives the required result.

Finally, combining Theorems 3.1 and 3.3 we have

\section{Theorem 3.4.}

$\pi(b, m, k)=m-\sum_{i=1}^{k} F_{b, m}\left(p_{i}\right)+\sum_{j=2}^{k} F_{b, m}\left(2 p_{j}\right)+\sum_{i=2}^{k-1} \sum_{j=i+1}^{k} \pi\left(c_{b}\left(p_{i} p_{j}\right), F_{b, m}\left(p_{i} p_{j}\right), i-1\right)$

for all $b, m$ and $k$.

\section{A LOWER BOUND ON $\pi_{\min }(m, k)$}

We now give a lower bound on $\pi_{\min }(m, k)$, the lowest value of $\pi(b, m, k)$ across all $b$. This bound makes use of constraints on the co-occurence of residues of primes to $P_{k}$. We begin with a very obvious result, which we give without proof.

Theorem 4.1. If $d \nmid m$, then

$$
F_{b, m}(d)=\left\lceil\frac{m}{d}\right\rceil \Leftrightarrow(b+m) \bmod d<m \bmod d .
$$

Using this we get

Theorem 4.2. For primes $p$ and $q$ let $x=m \bmod p$. If $x>0$ and $q \mid m-x+p$, then

$$
F_{b, m}(p)=\left\lceil\frac{m}{p}\right\rceil \Rightarrow F_{b, m}(p q)=\left\lceil\frac{m}{p q}\right\rceil .
$$


Proof. Let $x=m \bmod p$. Assume $x>0, q \mid m-x+p$ and

$$
F_{b, m}(p)=\left\lceil\frac{m}{p}\right\rceil \text {. }
$$

Then since $x>0$ means $p \nmid m$ from Theorem 4.1 we have

$$
(b+m) \bmod p<x .
$$

Let

$$
y=b+m-((b+m) \bmod p) .
$$

Then $p$ divides every integer in the arithmetic sequence $y, y-p, \ldots, y-(q-1) p$. Since there are $q$ terms in this sequence, one of these terms is also divided by $q$, and so

$$
(b+m) \bmod p q \leq b+m-y+(q-1) p .
$$

However,

$$
b+m-y=(b+m) \bmod p<x
$$

and so

$$
(b+m) \bmod p q<x+(q-1) p .
$$

By assumption we have $q \mid m-x+p$; by definition $p \mid m-x$ and so we have $p q \mid m-x+p$ and $x<p<p q$. We thus get $m \bmod p q=x-p+p q=x+(q-1) p$ and so

$$
(b+m) \bmod p q<m \bmod p q,
$$

and from Theorem 4.1 we get the required result.

Theorem 4.3. For any positive integers $k$ and $m$ there exists an integer $b$ such that $\pi(b, m, k)=\pi_{\min }(m, k)$ and such that for all odd primes $p \mid m-1$ we have

$$
F_{b, m}(p)=\left\lceil\frac{m}{p}\right\rceil \Rightarrow F_{b, m}(2 p)=\left\lceil\frac{m}{2 p}\right\rceil .
$$

Proof. Let $b$ be some integer such that $\pi(b, m, k)=\pi_{\min }(m, k)$ and $2 \mid b+m$. (To see that we will always be able to select such a $b$, note that for any $a$ such that $\pi(a, m, k)=\pi_{\text {min }}(m, k)$ we either have $2 \mid a+m$ or $2 \nmid a+m$. If $2 \mid a+m$, then $b=a$ satisfies our requirements, whereas if $2 \nmid a+m$ then $2 \mid a+m+1$ and so $\pi(a+1, m, k)=\pi(a, m, k)=\pi_{\min }(m, k)$ and $b=a+1$ satisfies our requirements.) For any $p \mid m-1$ we have

$$
F_{b, m}(p)=\left\lceil\frac{m}{p}\right\rceil \Rightarrow(b+m) \bmod p<m \bmod p=1
$$

(from Theorem 4.1) and so for any $p \mid m-1$ we have

$$
F_{b, m}(p)=\left\lceil\frac{m}{p}\right\rceil \Rightarrow p|(b+m) \Rightarrow 2 p|(b+m) \Rightarrow F_{b, m}(2 p)=\left\lceil\frac{m}{2 p}\right\rceil
$$

as required.

Combining these results we get the following 
Theorem 4.4. For integer $m$ let $r=m \bmod P_{k}$ and let

$$
E=\mid\left\{i: 1<i \leq k, p_{i} \nmid r, 2 \mid\left(r-\left(r \bmod p_{i}\right)+p_{i}\right) \text { or } p_{i} \mid r-1\right\} \mid .
$$

Then

$$
\begin{aligned}
\phi\left(P_{k}\right)\left\lfloor\frac{m}{P_{k}}\right\rfloor+r-\sum_{i=1}^{k}\left\lceil\frac{r}{p_{i}}\right\rceil & +\sum_{i=2}^{k}\left\lfloor\frac{r}{2 p_{i}}\right\rfloor+E \\
& +\sum_{i=2}^{k-1} \sum_{j=i+1}^{k} \pi_{\min }\left(\left\lfloor\frac{r}{p_{i} p_{j}}\right\rfloor, i-1\right) \leq \pi_{\min }(m, k)
\end{aligned}
$$

for all $m$ and $k$.

Proof. Assume $r$ and $E$ as defined above. Let $b$ be an integer for which the conditions in Theorem 4.3 hold. Since Euler's totient $\phi(n)$ gives the number of integers coprime to $n$ in any sequence of $n$ consecutive integers we have

$$
\pi(b, m, k)=\phi\left(P_{k}\right)\left\lfloor\frac{m}{P_{k}}\right\rfloor+\pi(b, r, k)
$$

and we need only consider the value of $\pi(b, r, k)$. From Theorems 4.2 and 4.3 we see that

$$
F_{b, r}(p)=\left\lceil\frac{r}{p}\right\rceil \text { and } F_{b, r}(2 p)=\left\lfloor\frac{r}{2 p}\right\rfloor
$$

cannot hold for any prime counted in the definition of $E$, and so we have

$$
r-\sum_{i=1}^{k}\left\lceil\frac{r}{p_{i}}\right\rceil+\sum_{i=2}^{k}\left\lfloor\frac{r}{2 p_{i}}\right\rfloor+E \leq r-\sum_{i=1}^{k} F_{b, r}\left(p_{i}\right)+\sum_{j=2}^{k} F_{b, r}\left(2 p_{i}\right) .
$$

Combining this with the fact that by definition

$$
\pi_{\min }\left(\left\lfloor\frac{m}{p_{i} p_{j}}\right\rfloor, i-1\right) \leq \pi\left(c_{b}\left(p_{i} p_{j}\right), F_{b, m}\left(p_{i} p_{j}\right), i-1\right)
$$

we get

$$
\begin{aligned}
\phi\left(P_{k}\right)\left\lfloor\frac{m}{P_{k}}\right\rfloor+r-\sum_{i=1}^{k}\left\lceil\frac{r}{p_{i}}\right\rfloor+\sum_{i=2}^{k}\left\lfloor\frac{r}{2 p_{i}}\right\rfloor+E+\sum_{i=2}^{k-1} \sum_{j=i+1}^{k} \pi_{m i n}\left(\left\lfloor\frac{r}{p_{i} p_{j}}\right\rfloor, i-1\right) \\
\leq m-\sum_{i=1}^{k} F_{b, m}\left(p_{i}\right)+\sum_{j=2}^{k} F_{b, m}\left(2 p_{j}\right)+\sum_{i=2}^{k-1} \sum_{j=i+1}^{k} \pi\left(c_{b}\left(p_{i} p_{j}\right), F_{b, m}\left(p_{i} p_{j}\right), i-1\right) .
\end{aligned}
$$

From Theorem 3.4 the right-hand side of this expression is equal to $\pi(b, m, k)$, which from Theorem 4.3 is equal to $\pi_{\min }(m, k)$ and we get the required result.

\section{Computations}

We first describe Algorithm 1, which computes a recursive function $\pi_{\text {low }}(m, k)$. Values of this function give a lower bound on $\pi_{\min }(m, k)$ as given in Theorem 4.4.

In this algorithm the variable $L$ is used to hold the incrementally computed lower bound on $\pi_{\min }(m, k)$. Lines 2 to 4 obtain a value for $\left\lfloor\frac{m}{P_{k}}\right\rfloor$, assign an initial value for $L$ as in Theorem 4.4, and obtain a value for $r$ (again as in Theorem 4.4).

We explicitly computed the value of $\pi_{\min }(m, k)$ for all $k$ less than or equal to 6 and for all $m$ less than $P_{k}$; these provide stopping conditions for the recursion in $\pi_{\text {low }}(m, k)$ (Line 5). 
Algorithm 1. The function $\pi_{\text {low }}(m, k)$. This function requires that for all $k \leq 6$, values of $\pi_{m i n}(m, k)$ are known for all $m \leq P_{k}$. Known values of $h(k)$ for $k$ less than 50 are used only if the variable UseKnown is set.

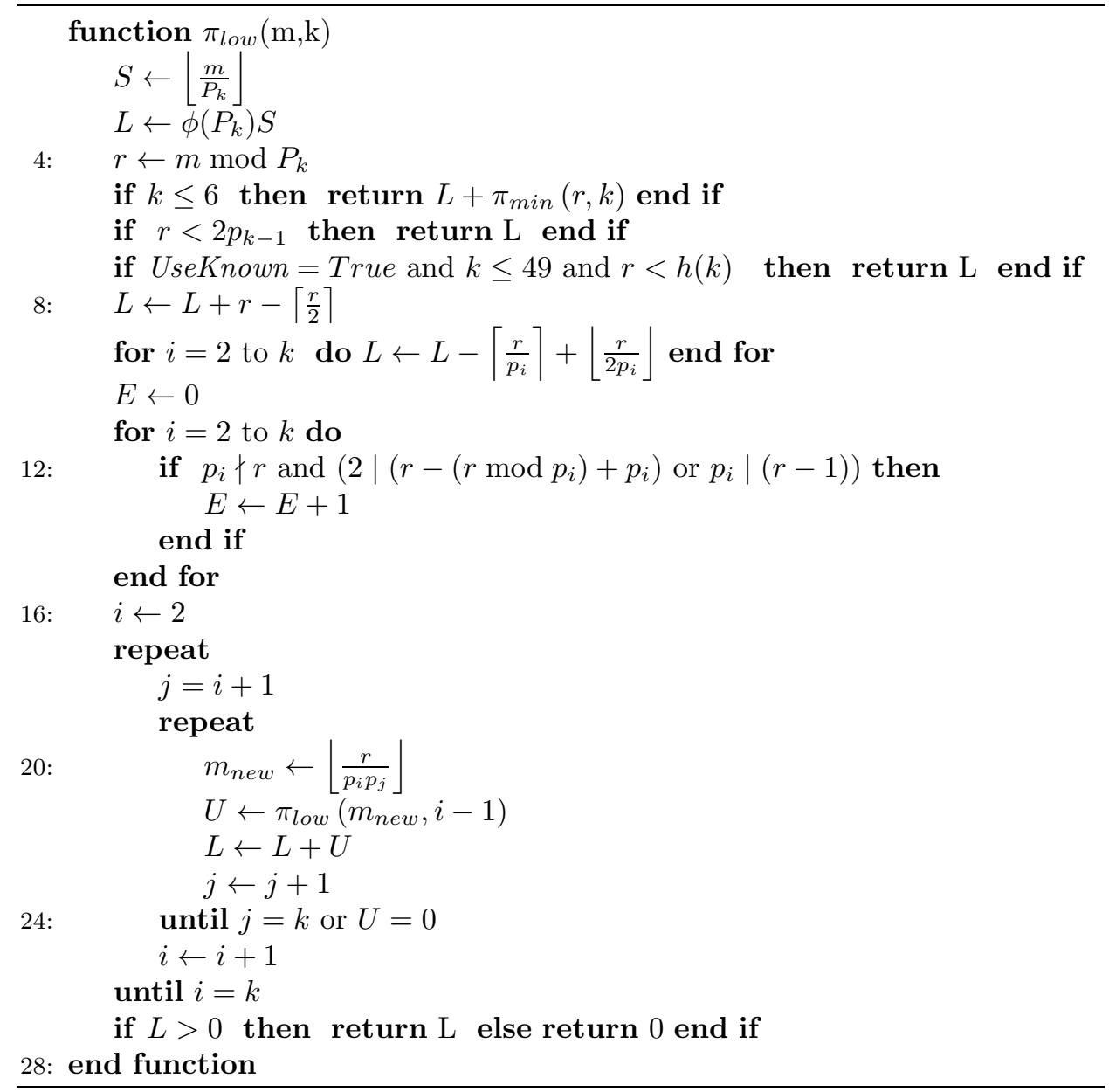

From Hagedorn [2] we have explicit values of $h(k)$ for $k \leq 49$, and we also have the general result that $2 p_{k-1} \leq h(k)$ for all $k$. Since $\pi_{\min }(m, k)=0$ if $m<h(k)$ we use both the explicit values and the general result as further stopping conditions for the recursion (Lines 6 and 7). Hagedorn's explicit values for $h(k)$ for $k \leq 49$ are used only if the variable UseKnown is set to true.

Lines 8 and 9 calculate the first three terms in the left-hand side of Theorem 4.4, with Lines 10 to 15 calculating the $E$ term in that sum.

Lines 16 to 26 give two nested loops containing recursive calls to $\pi_{l o w}(\cdot)$ and so calculating a lower bound on value of the double sum in Theorem 4.4. For each pair of values for indices $i$ and $j$ in these loops, the algorithm recursively gets a lower bound on $\pi_{\text {min }}\left(m_{n e w}, i-1\right)$ as in Theorem 4.4 placing this bound in a variable $U$.

Finally, line 27 returns a lower bound on $\pi_{\min }(m, k)$. Since $\pi_{\min }(m, k)$ cannot be less than 0 (no sequence contains a negative number of primes) the algorithm returns the computed bound $L$ only if $L>0$; otherwise 0 is returned. 

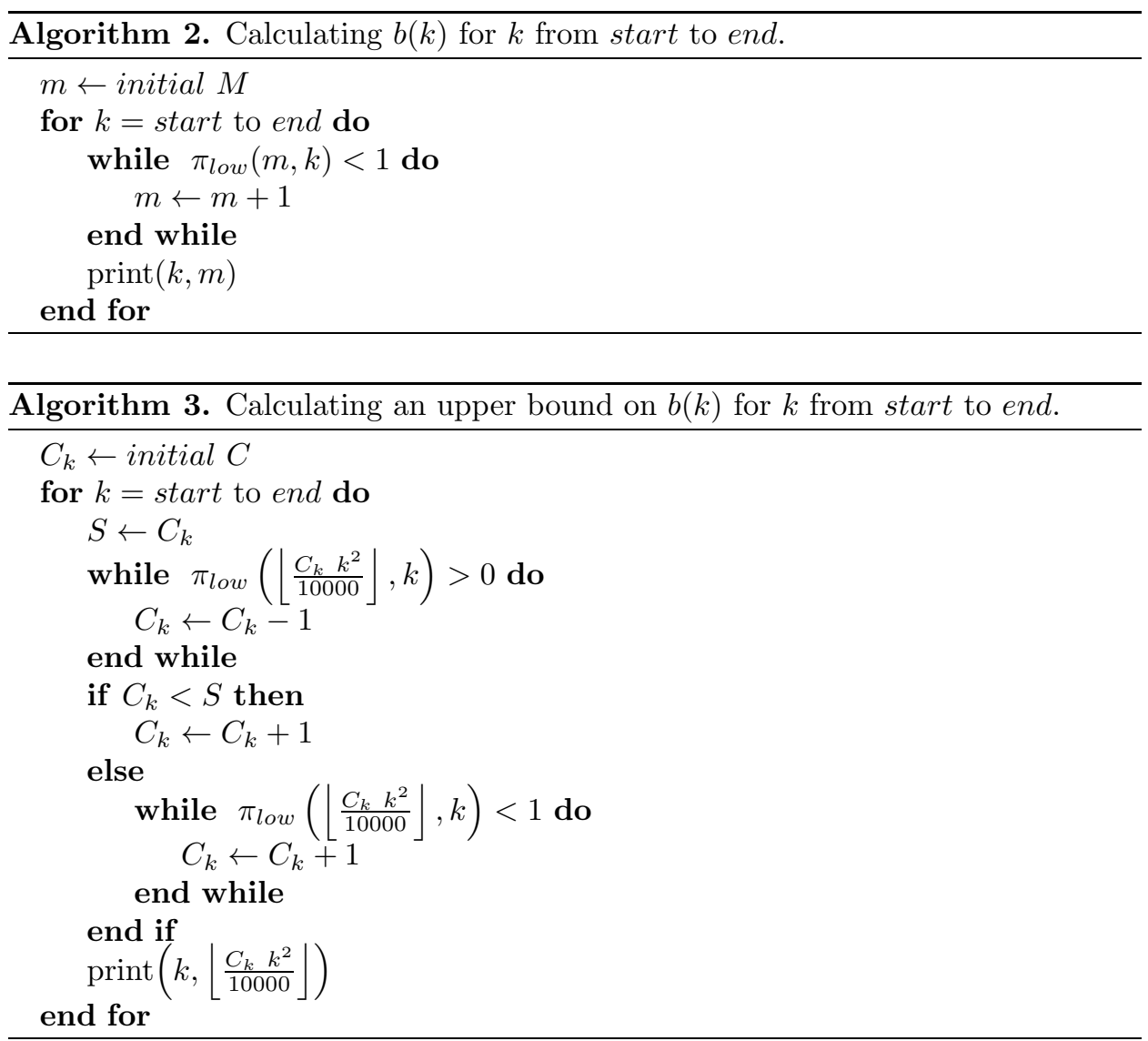

At Lines 24 and 26 the inner and outer loops terminate at $j=k$ and $i=k-1$ respectively, as in the double sum in Theorem 4.4. For efficiency the inner loop also terminates if the last obtained recursive lower bound value $U$ was equal to 0 ; in this case all further recursive calls within this loop will also return a value of 0 , because $m_{n e w}$ is decreasing on each cycle of the inner loop. Similarly, the outer loop terminates if the first recursive bound value $U$ obtained on the previous cycle of the inner loop had a value of 0 ; in this case recursive calls in all further cycles of the inner loop will also return a value of 0 , because all values of $m_{n e w}$ in those further cycles will be less than the current value of $m_{\text {new }}$.

We implemented the function $\pi_{\text {low }}$ in the computer algebra system PARI [1]. We take $b(k)$ to represent the lowest integer $m$ such that $\pi_{l o w}(m, k)>0$ and so $b(k)$ is an upper bound on $h(k)$. We find $b(k)$ using linear search across increasing values of $m$ (Algorithm 2).

To compare values of $b(k)$ with the values of $h(k)$ calculated by Hagedorn for $k$ from 1 to 49 [2] we ran Algorithm 2 with start $=1$, end $=49$, initial $M=1$ and variable UseKnown set to False. In this range the bound $b(k)$ was less than 3 times the true value of $h(k)$. Figure 1 graphs $h(k), b(k)$, and Stevens' and Kanold's bounds on $h(k)$ in this range.

To calculate values of $b(k)$ up to $k=1000$ we ran Algorithm 2 with start $=50$, end $=1000$, variable UseKnown set to True, and initial $M$ set to $h(49)=742$. 


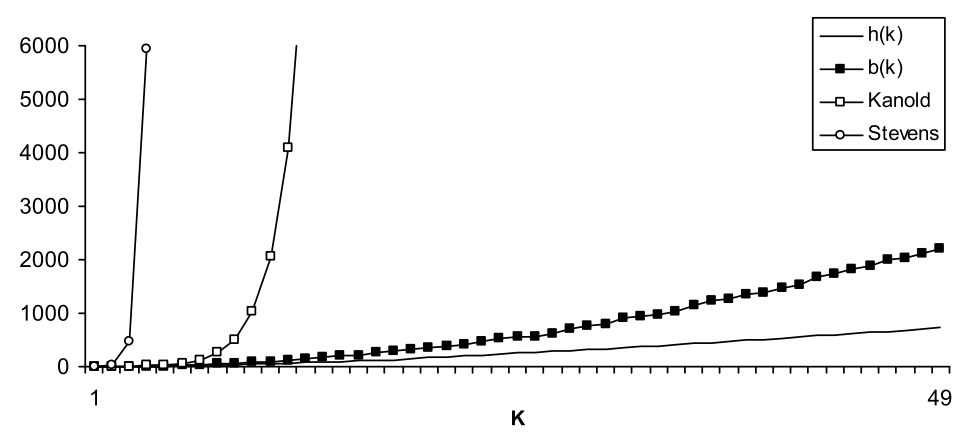

Figure 1. Graph comparing $h(k), b(k)$, Kanold's bound and Stevens' bound for $k$ up to 49 .

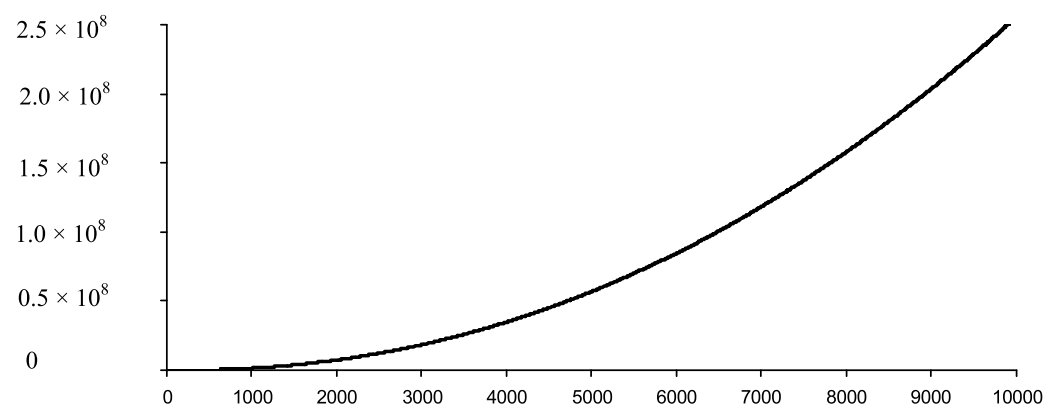

FiguRE 2. Graph of $b(k)$ vs. $k$ for $k$ from 50 to 10000

For a given $k$ Algorithm 2 calculates values of $\pi_{l o w}(m, k)$ for increasing values of $m$ until $b(k)$ is reached (that is, until $\left.\pi_{l o w}(m, k)>0\right)$. Clearly the number of integers $m$ for which Algorithm 2 must calculate $\pi_{l o w}(m, k)$ grows as $k$ grows. Algorithm 2 will thus be relatively slow for large $k$ (in our runs Algorithm 2 took around 15 minutes to calculate $b(1000)$ ). For this reason when calculating bounds $b(k)$ for $k$ greater than 1000 we took an alternate approach, using the function $\pi_{\text {low }}$ to find the lowest integer $C_{k}$ such that

$$
\pi_{\text {low }}\left(\left\lfloor\frac{C_{k} k^{2}}{10000}\right\rfloor, k\right)>0
$$

(see Algorithm 3). For each $C_{k}$ we then have $h(k) \leq\left(C_{k} k^{2}\right) / 10000$. To calculate values of $b(k)$ up to $k=10000$ we ran Algorithm 3 with start $=1001$, end $=10000$, variable UseKnown set to True, and initial $C$ set to 10000.

Figure 2 graphs $b(k)$ for $k$ from 50 to 10000 as obtained from these two algorithms. We find

$$
b(k) \leq 0.27749612254 k^{2} \log k
$$

for all $k$ in this range, and so this gives an upper bound on $h(k)$ for these $k$.

Figure 3 compares the logs of $b(k)$, Stevens' bound, and Kanold's bounds for $k$ up to 10000 . From this graph we see that $b(k)$ is hundreds of orders of magnitude stronger than Stevens' and Kanold's bounds in this range. 


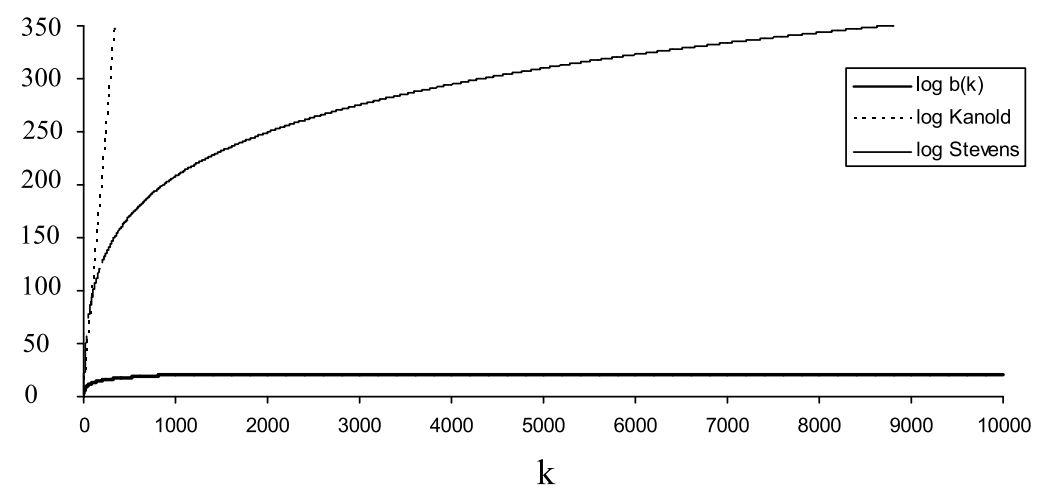

FiguRE 3. Graph comparing the log of $b(k)$ with the logs of Kanold's and Stevens' bounds for $k$ to 10000.

\section{REFERENCES}

[1] P. Erdős, On the integers relatively prime to $n$ and on a number-theoretic function considered by Jacobsthal, Math. Scand. 10 (1962), 163-170. MR0146125 (26 \#3651)

[2] Thomas R. Hagedorn, Computation of Jacobsthal's function $h(n)$ for $n<50$, Math. Comp. 78 (2009), no. 266, 1073-1087, DOI 10.1090/S0025-5718-08-02166-2. MR2476571 (2009k:11146)

[3] Lajos Hajdu and Natarajan Saradha, On a problem of Pillai and its generalizations, Acta Arith. 144 (2010), 323-347.

[4] L. Hajdu and N. Saradha, Disproof of a conjecture of Jacobsthal, Math. Comp. 81 (2012), no. 280, 2461-2471, DOI 10.1090/S0025-5718-2012-02581-6. MR2945166

[5] Henryk Iwaniec, On the problem of Jacobsthal, Demonstratio Math. 11 (1978), no. 1, 225-231. MR 499895 (80h:10053)

[6] Hans-Joachim Kanold, Über eine zahlentheoretische Funktion von Jacobsthal (German), Math. Ann. 170 (1967), 314-326. MR0209247 (35 \#149)

[7] Helmut Maier and Carl Pomerance, Unusually large gaps between consecutive primes, Trans. Amer. Math. Soc. 322 (1990), no. 1, 201-237, DOI 10.2307/2001529. MR972703 (91b:11093)

[8] János Pintz, Very large gaps between consecutive primes, J. Number Theory 63 (1997), no. 2, 286-301, DOI 10.1006/jnth.1997.2081. MR.1443763 (98c:11092)

[9] Carl Pomerance, A note on the least prime in an arithmetic progression, J. Number Theory 12 (1980), no. 2, 218-223, DOI 10.1016/0022-314X(80)90056-6. MR578815 (81m:10081)

[10] Harlan Stevens, On Jacobsthal's g(n)-function, Math. Ann. 226 (1977), no. 1, 95-97. MR0427212 (55 \#247)

[11] The PARI Group, Bordeaux, PARI/GP, version 2.5.3, 2012, available from http://pari. math.u-bordeaux.fr/.

School of Computer Science and Informatics, University College Dublin, Belfield, Dublin 6, IRELAND

E-mail address: fintan.costello@ucd.ie

Department of Mathematical Physics, National University of Ireland Maynooth, Maynooth, Co. Kildare, Ireland

E-mail address: watts@thphys.nuim.ie 